\title{
Case Report \\ Parotid Oncocytoma as a Manifestation of Birt-Hogg-Dubé Syndrome
}

\author{
Kazuki Yoshida ${ }^{D},{ }^{1}$ Masao Miyagawa, ${ }^{1}$ Teruhito Kido, ${ }^{1}$ Kana Ide, \\ Yoshifumi Sano, ${ }^{2}$ Yoshifumi Sugawara, ${ }^{3}$ Hiroyuki Takahata, ${ }^{4}$ Nobuya Monden, ${ }^{5}$ \\ Mitsuko Furuya, ${ }^{6}$ and Teruhito Mochizuki ${ }^{1}$ \\ ${ }^{1}$ Department of Radiology, Ehime University Graduate School of Medicine, Toon, Japan \\ ${ }^{2}$ Department of Pulmonary Surgery, Ehime University Graduate School of Medicine, Toon, Japan \\ ${ }^{3}$ Department of Diagnostic Radiology, Shikoku Cancer Center, Matsuyama, Japan \\ ${ }^{4}$ Department of Pathology, Shikoku Cancer Center, Matsuyama, Japan \\ ${ }^{5}$ Department of Head and Neck Surgery, Shikoku Cancer Center, Matsuyama, Japan \\ ${ }^{6}$ Department of Molecular Pathology, Yokohama City University Graduate School of Medicine, Yokohama, Japan
}

Correspondence should be addressed to Kazuki Yoshida; kn0wn951753@gmail.com

Received 8 January 2018; Accepted 7 May 2018; Published 3 June 2018

Academic Editor: Bruce J. Barron

Copyright (C) 2018 Kazuki Yoshida et al. This is an open access article distributed under the Creative Commons Attribution License, which permits unrestricted use, distribution, and reproduction in any medium, provided the original work is properly cited.

Birt-Hogg-Dubé syndrome (BHD) is a rare autosomal dominant disease characterized by skin fibrofolliculomas, pulmonary cysts, spontaneous pneumothoraces, and renal cancers. Oncocytomas are benign epithelial tumors that are also rare. Recently, there have been a few case reports of BHD with a parotid oncocytoma that appears to have a BHD phenotype. Here we document the eighth known case and describe the magnetic resonance imaging features of the parotid oncocytoma, which mimicked Warthin's tumor. Radiologists should be aware of the association between these rare disorders.

\section{Introduction}

Birt-Hogg-Dubé syndrome (BHD) is a rare autosomal dominant disease characterized by skin fibrofolliculomas, pulmonary cysts, spontaneous pneumothoraces, and renal cancers [1]. In 1977, Birt, Hogg, and Dubé reported on a group of patients from single kindred who had multiple fibrofolliculomas with trichodiscomas and acrochordons [2]. This hereditary condition was later named Birt-Hogg-Dubé syndrome. In 2002, Nickerson et al. identified the BHD gene, which codes a protein called folliculin [3]. The BHD gene is now known as the folliculin gene (FLCN).

Patients with BHD often have renal tumors. Pavlovich et al. reported that $34(27 \%)$ of 124 patients with BHD had renal tumors with variable histology, most commonly hybrid oncocytic tumors, and chromophobe renal cell carcinomas [4]. However, there are few reported cases of BHD with parotid gland oncocytoma. Here we present a rare case of a patient with this association.

\section{Case History}

A 44-year-old woman presented to our hospital complaining of right lower facial swelling and pain around the parotid gland. Her past medical history was unremarkable. However, she had a family history of pneumothoraces in her father and brother. Magnetic resonance (MR) imaging showed a $35 \mathrm{~mm}$ diameter mass in the superficial lobule of the right parotid gland. The lesion appeared hypointense on both T1-weighted MR imaging (T1WI) and T2-weighted imaging (T2WI). On fat-saturated T2WI, the masses appeared hyperintense when compared with the native parotid gland tissue but hypointense on contrast-enhanced T1WI with fat saturation. The lesion was hyperintense on axial diffusion-weighted imaging with a b-value of $1000 \mathrm{~s} / \mathrm{mm}^{2}$ and a low apparent diffusion coefficient (Figure 1). The lesion was suspected to be Warthin's tumor, and a right superficial parotidectomy was performed accordingly. 


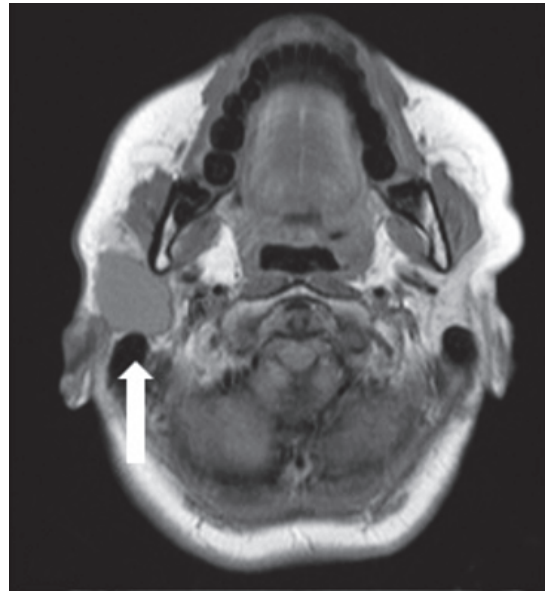

(a)

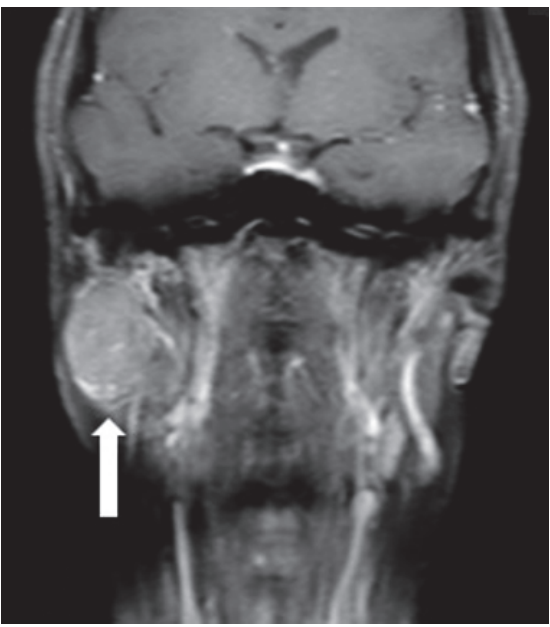

(d)

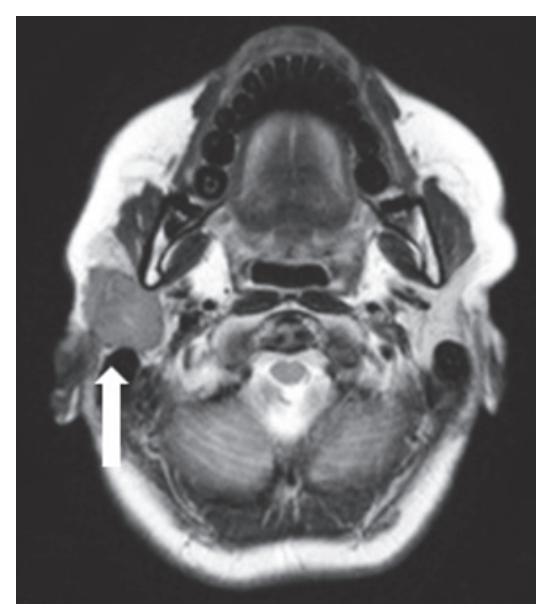

(b)

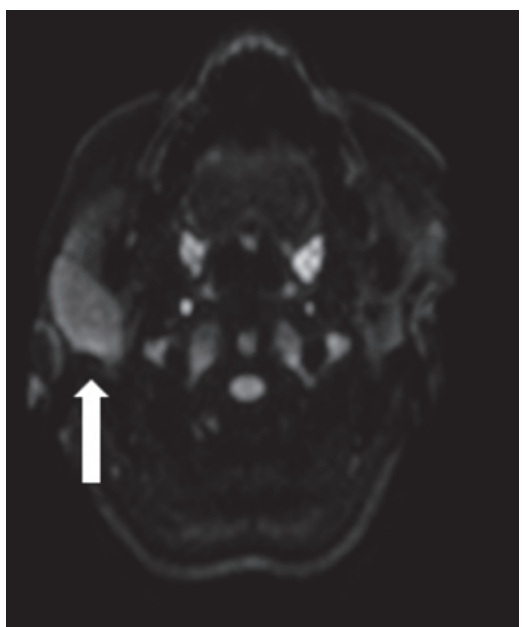

(e)

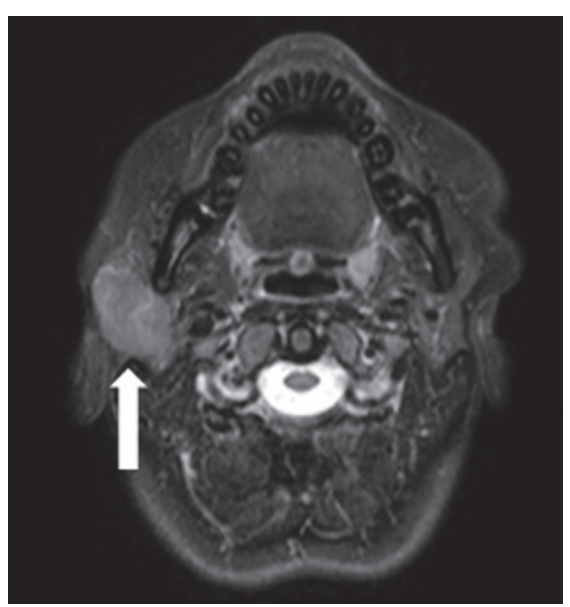

(c)

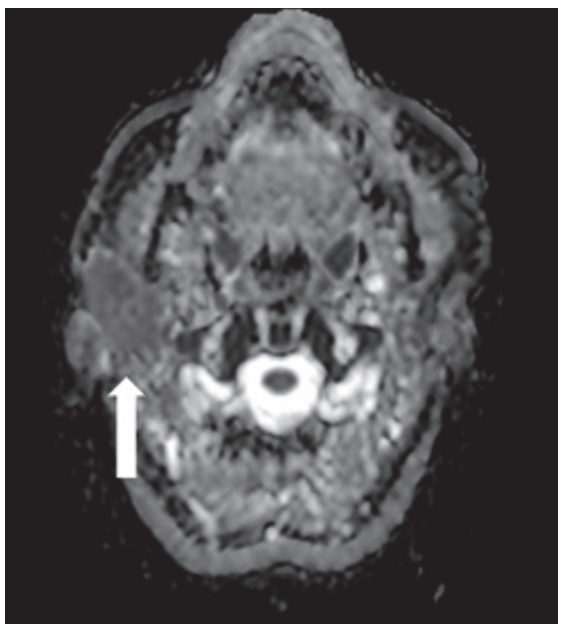

(f)

Figure 1: Magnetic resonance images of parotid oncocytoma. (a) T1-weighted imaging. (b) T2-weighted imaging. An axial magnetic resonance image shows a well-demarcated mass (white arrow) in the superficial and deep lobe of the right parotid gland. (c, d) The mass appears hyperintense to the native parotid gland tissue on fat-saturated T2-weighted imaging but hypointense on contrast-enhanced, fatsaturated T1-weighted imaging. (e, f) On diffusion-weighted images, the mass appeared hyperintense and hypointense according to the apparent diffusion coefficient.

There was a well-circumscribed, solid, mahogany-colored nodule measuring $3.7 \times 2.8 \times 2.4 \mathrm{~cm}$ in the superficial parotidectomy specimen (Figure 2(a)). Microscopically, the nodule was an encapsulated tumor containing oncocytic cells. These cells formed solid clusters or trabecular patterns, separated by thin strands of fibrovascular stroma (Figure 2(b)) and were round in shape with centrally placed nuclei and clear cytoplasm. Neither necrosis nor capsular invasion was observed. Cytoplasmic granules enriched with glycogen were present but there was no mucin on periodic acidSchiff staining (data not shown). Although the morphology was comparable to oncocytoma of the kidney, radiologic examination excluded the possibility of a metastatic renal tumor, and immunostaining for PAX-2 and CD10, which are markers for renal cell carcinoma, was negative (data not shown). The pathologic diagnosis was clear cell oncocytoma.

One and a half years later, the patient presented to hospital again, this time with mild dyspnea. Physical examination revealed decreased breath sounds on the right side. A computed tomography (CT) scan showed a right pneumothorax and multiple cysts. The cysts were located in the medial basilar regions of the lung fields bilaterally and were ellipsoid in shape and variable in size. Some of the cysts abutted the proximal portions of the lower pulmonary arteries (Figure 3 ). The patient was strongly suspected to have BHD and was subsequently referred for genetic counseling. Informed consent was obtained from the patient for FLCN genetic testing, which was performed on genomic DNA extracted from 


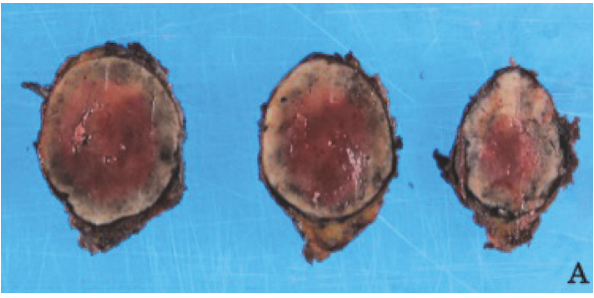

(a)

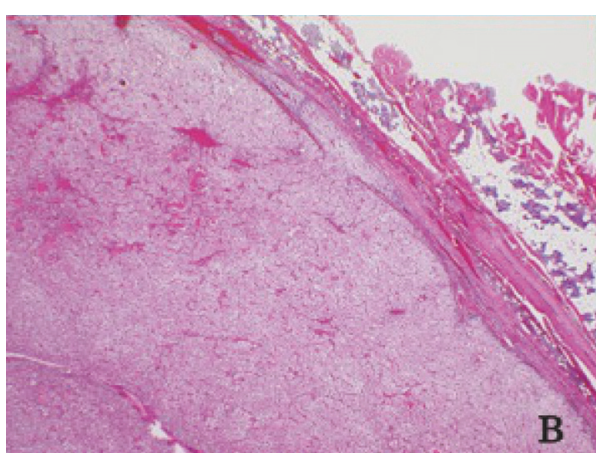

(b)

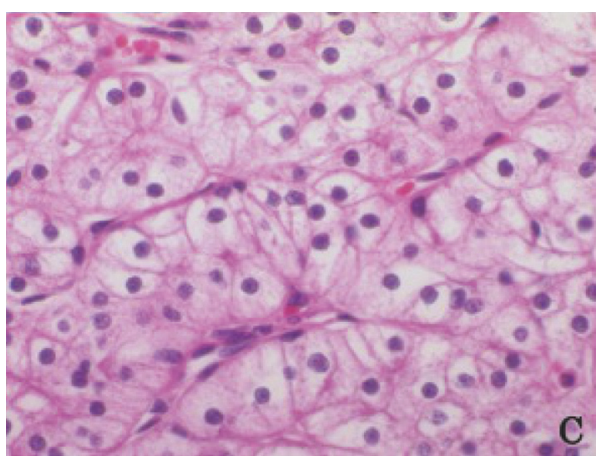

(c)

Figure 2: Histopathologic findings of parotid clear cell oncocytoma. (a) A well-circumscribed, solid, mahogany-colored nodule. (b, c) Microscopically, fibrous encapsulated nodules containing oncocytic tumor cells with a clear cytoplasm. (hematoxylin-eosin staining; original magnification, $20 \times$ in (b) and $400 \times$ in (c))

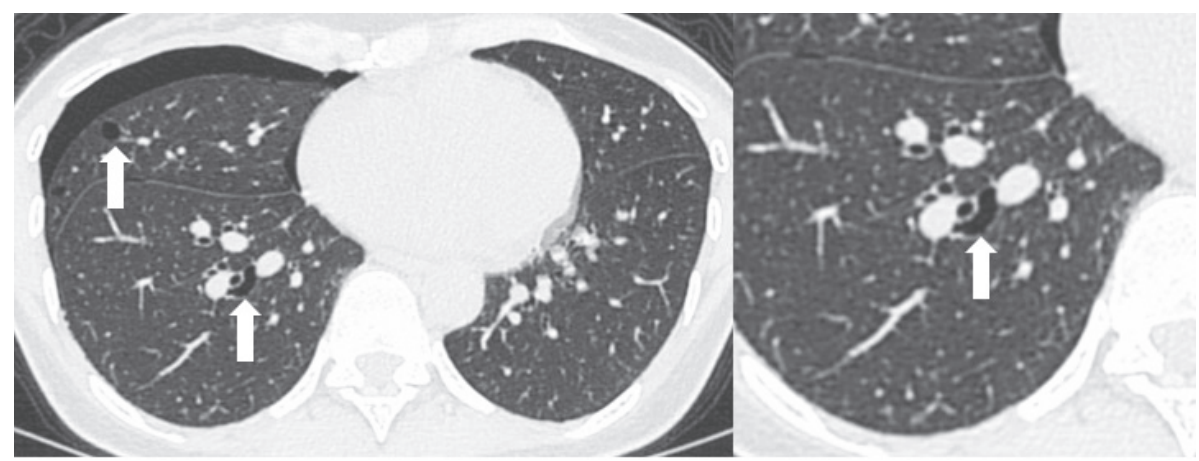

Figure 3: Pulmonary computed tomography demonstrating a right pneumothorax and multiple cysts (arrow). An ellipsoidal cyst abuts on the proximal portion of the pulmonary artery.

peripheral leukocytes. Duplication of cytosine was identified in the C8 tract of exon 11 (c.1285dupC), confirming the diagnosis of BHD. The patient's brother, who had an episode of pneumothorax, asked for genetic testing and was found to have the same mutation as the proband (data not shown).

\section{Discussion}

BHD is a rare disease and there are some reports of its prevalence. In North America, 102 BHD-affected families have been reported by the National Cancer Institute group 
[6, 15]. In Asia, 312 affected individuals from 120 Japanese BHD families have been reported [16]. However, information regarding the manifestations of BHD apart from pulmonary cysts, renal tumors, and cutaneous manifestations is limited. Oncocytomas are rare benign epithelial tumors, accounting for only $0.5 \%-1.5 \%$ of all salivary gland tumors. The parotid gland is the site most often affected, accounting for $78 \%-84 \%$ of salivary gland oncocytomas [12]. These tumors are slightly more prevalent in women than in men and usually occur in the seventh to ninth decades of life [14].

Lieu et al. reported the first case of BHD with parotid oncocytoma in a 56-year-old man [5]. Lindor et al. then reported a 45-year-old Caucasian woman with BHD who presented with multiple oncocytic parotid tumors [8]. Pradella et al. also reported a parotid oncocytoma that had arisen in a patient with BHD [9]. In a report by Schmidt et al. in 2005 on 219 BHD-affected individuals, four parotid gland tumors were documented in 2 men and 2 women. Three of those tumors were classified as oncocytoma [6]. In 2011, Maffé et al. reported on 19 BHD-affected individuals, including a 53-year-old man with bilateral parotid oncocytoma. There was a relative reduction of the wild-type signal from the parotid oncocytoma in this patient, who was heterozygous for the FLCN mutation. They reported that parotid oncocytoma should be considered as a component manifestation of BHD [7]. To the best of our knowledge, our patient is the eighth reported case of BND with parotid oncocytoma. The previous seven reports and our present case are summarized in Table 1.

FLCN encodes the protein folliculin (FLCN), which acts as a tumor suppressor. FLCN forms a complex with FLCNinteracting protein 1 (FNIP1) and FNIP1 homologue FNIP2, which interacts with 5'AMP-activated protein kinase and regulates signaling of the mammalian target of rapamycin $[17,18]$.

The clinical characteristics of BHD are thought to be age-related. Skin lesions usually develop after the age of 20 years. Pulmonary cysts and pneumothoraces are often found in young adult patients aged 20-30 years, and renal cell tumors are more likely to develop after the age of 40 years. Therefore, BHD should be considered in patients who develop repeated spontaneous pneumothoraces and have skin fibrofolliculomas [1, 17]. Abdominal imaging is recommended at least every 3 years in the clinical follow-up of BHD. We prefer MR imaging to CT as it avoids exposure to radiation [18].

The MR imaging reports on parotid oncocytomas suggest that these lesions are hypointense on both T1WI and T2WI $[10,14]$, are hyperintense on diffusion-weighted imaging, and have low apparent diffusion coefficient values. Dynamic contrast-enhanced MR images show early enhancement with early washout [10]. Parotid oncocytomas also show high uptake of ${ }^{18} \mathrm{~F}$-fluorodeoxyglucose on positron emission tomography (Table 2) [11]. Oncocytomas and Warthin's tumors have very similar features on imaging and therefore are very difficult to differentiate [19]. However, oncocytomas have unique imaging features that have led to them being known as "vanishing tumors" and may help in the diagnosis. Oncocytomas are hard to detect on fat-saturated T2 and $\mathrm{T} 1$ postcontrast MR images because of the similarity of intensity of the tumor and the parenchyma, hence the term vanishing tumor. Oncocytes have a low free water content and a lipid-rich membrane. If the amount of lipids in the tumor is approximately equal to that in the normal parotid gland tissue, the vanishing phenomenon occurs [13]. However, in our patient, the lesion appeared slightly hyperintense on fat-saturated T2WI and hypointense on contrast-enhanced and fat-saturated T1WI. The histology was that of a clear cell oncocytoma, which contains large amounts of glycogen and lipid that is different from that of normal oncocytes. A mixed oncocytoma/pleomorphic adenoma is hyperintense on fat-saturated T2WI and contrastenhanced, fat-saturated T1WI [13]. These different MR imaging features may reflect the histopathologic findings. The previous seven case reports did not describe the imaging findings in detail. It is uncertain if the MR findings of solitary oncocytoma are different from those of oncocytoma associated with BHD; therefore, further investigation is needed.

Tobino et al. reported that multiple, irregular-shaped cysts of various sizes with lower medial lung zone predominance were characteristic features of BHD on CT [20]. Cysts abutting or including the proximal portion of the lower pulmonary arteries or veins are also highly probable in BHD (Figure 3). Our patient had these features, which were very helpful in making a diagnosis of BHD.

In conclusion, we describe the eighth confirmed case in the literature of parotid oncocytoma in BHD, which mimicked Warthin's tumor on MR imaging. Parotid oncocytoma appears to be one of the phenotypes in BHD. It is difficult to distinguish between oncocytomas and Warthin's tumors; however, radiologists should be aware of this association and consider parotid oncocytoma as a differential diagnosis if they detect a parotid mass similar to Warthin's tumor in BHD. If additional imaging is to be recommended, a dedicated renal-protocol MRI would be the choice, alone or in addition to chest CT.

\section{Consent}

Informed consent to publish this report was obtained from the patient.

\section{Conflicts of Interest}

The authors declare that there are no conflicts of interest regarding the publication of this article. 


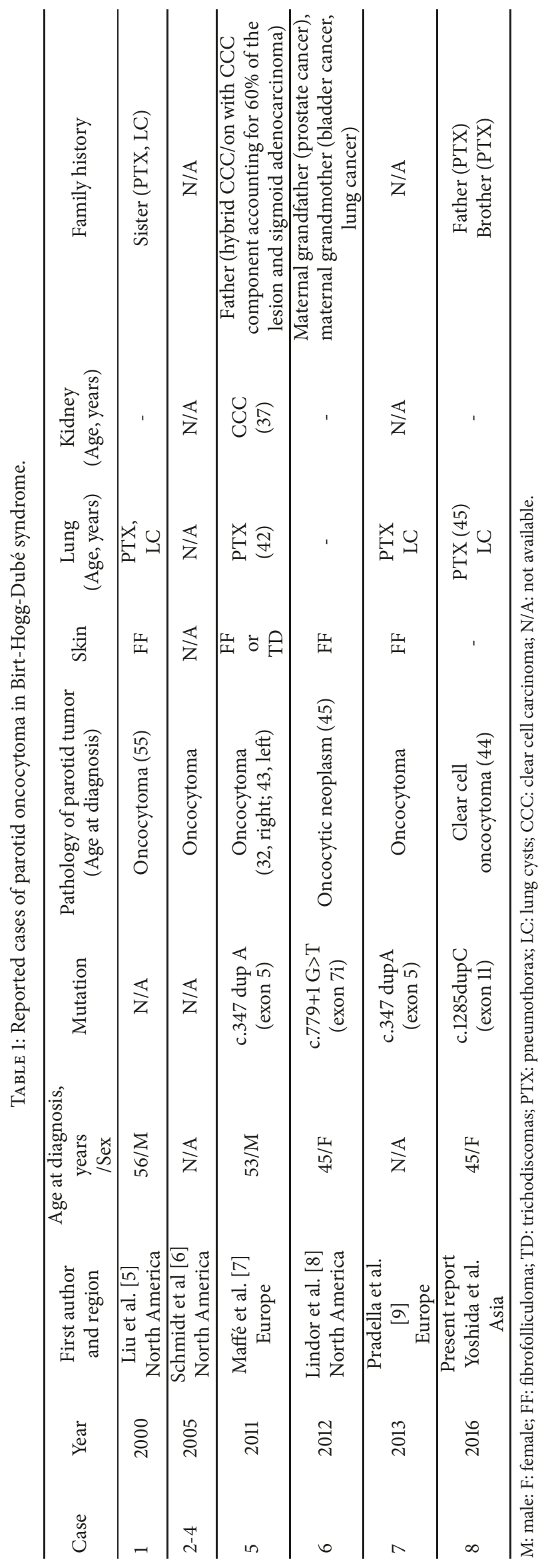




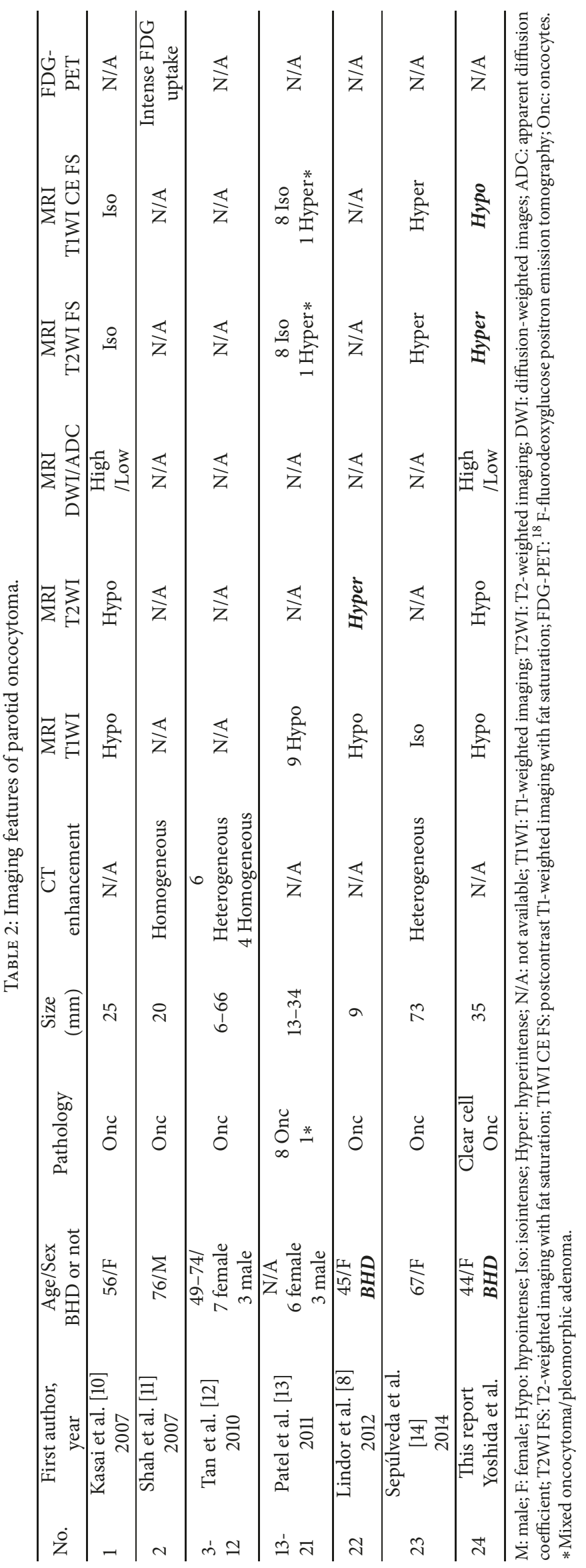




\section{Acknowledgments}

The authors thank Dr. Shojirou Morinaga, Department of Pathology, Hino Municipal Hospital, Hino, Japan, for making the pathological diagnosis in this patient.

\section{References}

[1] F. H. Menko, M. A. van Steensel, S. Giraud et al., "BirtHogg-Dubé syndrome: diagnosis and management," The Lancet Oncology, vol. 10, no. 12, pp. 1199-1206, 2009.

[2] A. R. Birt, G. R. Hogg, and W. J. Dube, "Hereditary multiple fibrofolliculomas with trichodiscomas and acrochordons," JAMA Dermatology, vol. 113, no. 12, pp. 1674-1677, 1977.

[3] M. L. Nickerson, M. B. Warren, J. R. Toro et al., "Mutations in a novel gene lead to kidney tumors, lung wall defects, and benign tumors of the hair follicle in patients with the Birt-Hogg-Dubé syndrome," Cancer Cell, vol. 2, no. 2, pp. 157-164, 2002.

[4] C. P. Pavlovich, R. L. Grubb III, K. Hurley et al., "Evaluation and management of renal tumors in the Birt-Hogg-Dubé syndrome," The Journal of Urology, vol. 173, no. 5, pp. 1482-1486, 2005.

[5] V. Liu, T. Kwan, and E. H. Page, "Parotid oncocytoma in the Birt-Hogg-Dubé syndrome," Journal of the American Academy of Dermatology, vol. 43, no. 6, pp. 1120-1122, 2000.

[6] L. S. Schmidt, M. L. Nickerson, M. B. Warren et al., "Germline BHD-mutation spectrum and phenotype analysis of a large cohort of families with Birt-Hogg-Dubé syndrome," American Journal of Human Genetics, vol. 76, no. 6, pp. 1023-1033, 2005.

[7] A. Maffé, B. Toschi, G. Circo et al., "Constitutional FLCN mutations in patients with suspected Birt-Hogg-Dubé syndrome ascertained for non-cutaneous manifestations," Clinical Genetics, vol. 79, no. 4, pp. 345-354, 2011.

[8] N. M. Lindor, J. Kasperbauer, J. E. Lewis, and M. Pittelkow, "Birt-Hogg-Dubé syndrome presenting as multiple oncocytic parotid tumors," Hereditary Cancer in Clinical Practice, vol. 10, no. 1, article 13, 2012.

[9] L. M. Pradella, M. Lang, I. Kurelac et al., "Where Birt-HoggDubé meets Cowden Syndrome: Mirrored genetic defects in two cases of syndromic oncocytic tumours," European Journal of Human Genetics, vol. 21, no. 10, pp. 1169-1172, 2013.

[10] T. Kasai, K. Motoori, T. Hanazawa, Y. Nagai, and H. Ito, "MR imaging of multinodular bilateral oncocytoma of the parotid gland," European Journal of Radiology Extra, vol. 63, no. 3, pp. 97-100, 2007.

[11] V. N. Shah and B. F. Branstetter, "Oncocytoma of the parotid gland: a potential false-positive finding on 18F-FDG PET," American Journal of Roentgenology, vol. 189, no. 4, pp. 212-214, 2007.

[12] T. J. Tan and T. Y. Tan, "CT features of parotid gland oncocytomas: A study of 10 cases and literature review," American Journal of Neuroradiology, vol. 31, no. 8, pp. 1413-1417, 2010.

[13] N. D. Patel, A. Van Zante, D. W. Eisele, H. R. Harnsberger, and C. M. Glastonbury, "Oncocytoma: The vanishing parotid mass," American Journal of Neuroradiology, vol. 32, no. 9, pp. 17031706, 2011.

[14] I. Sepúlveda, E. Platín, M. L. Spencer et al., "Oncocytoma of the parotid gland:a case report and review of the literature," Case Reports in Oncology, vol. 7, no. 1, pp. 109-116, 2014.
[15] J. R. Toro, M.-H. Wei, and G. M. Glenn, "BHD mutations, clinical and molecular genetic investigations of Birt-HoggDubé syndrome: a new series of 50 families and a review of published reports," Journal of Medical Genetics, vol. 45, no. 6, pp. 321-331, 2008.

[16] M. Furuya, M. Yao, R. Tanaka et al., "Genetic, epidemiologic and clinicopathologic studies of Japanese Asian patients with Birt-Hogg-Dubé syndrome," Clinical Genetics, vol. 90, no. 5, pp. 403-412, 2016.

[17] M. Furuya and Y. Nakatani, "Birt-Hogg-Dubé syndrome: clinicopathological features of the lung," Journal of Clinical Pathology, vol. 66, no. 3, pp. 178-186, 2013.

[18] L. Stamatakis, A. R. Metwalli, L. A. Middelton, and W. Marston Linehan, "Diagnosis and management of BHD-associated kidney cancer," Familial Cancer, vol. 12, no. 3, pp. 397-402, 2013.

[19] Y. Araki and R. Sakaguchi, "Synchronous oncocytoma and Warthin's tumor in the ipsilateral parotid gland," Auris Nasus Larynx, vol. 31, no. 1, pp. 73-78, 2004.

[20] K. Tobino, Y. Gunji, M. Kurihara et al., "Characteristics of pulmonary cysts in Birt-Hogg-Dubé syndrome: Thin-section CT findings of the chest in 12 patients," European Journal of Radiology, vol. 77, no. 3, pp. 403-409, 2011. 


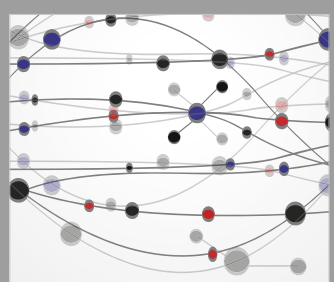

The Scientific World Journal
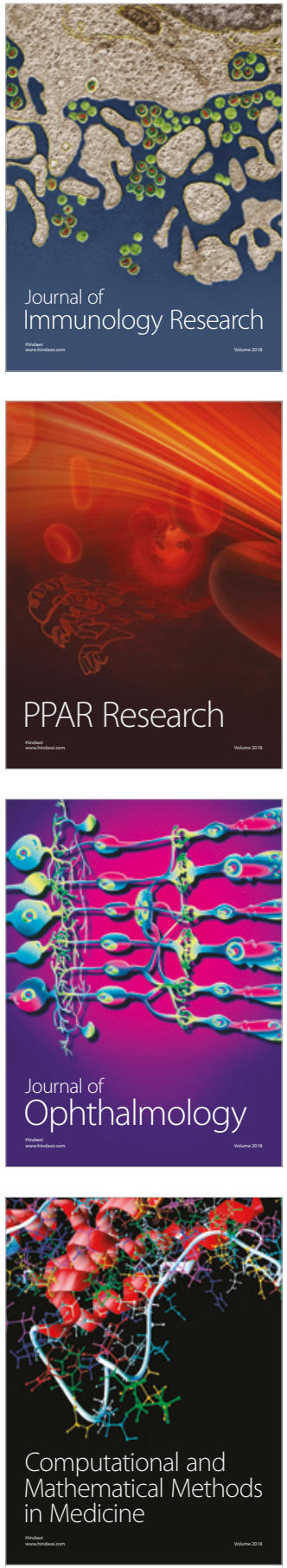

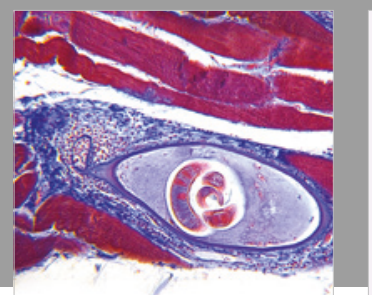

Gastroenterology Research and Practice

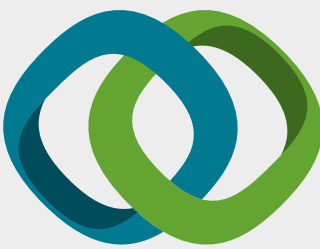

\section{Hindawi}

Submit your manuscripts at

www.hindawi.com
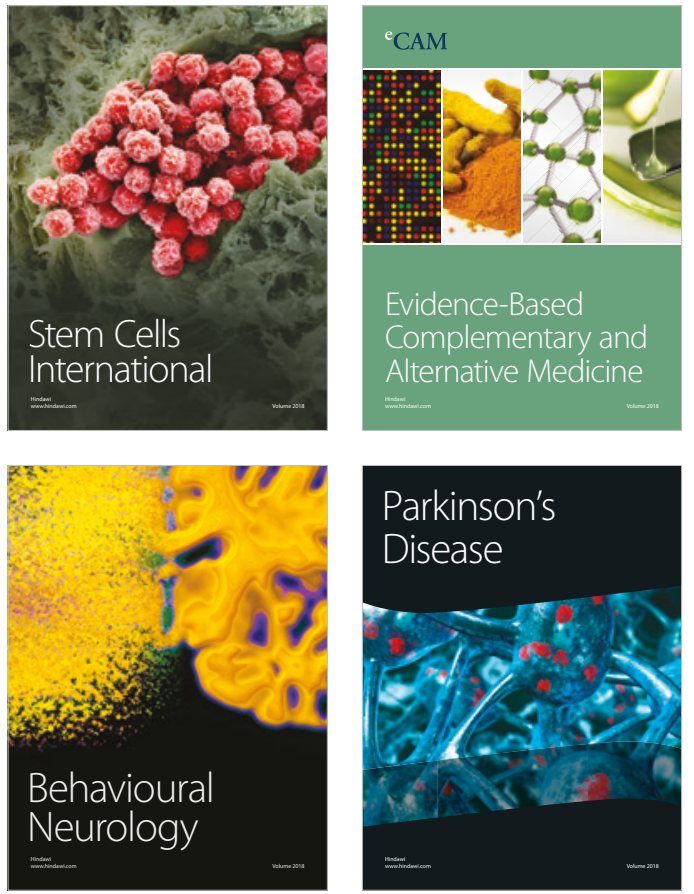

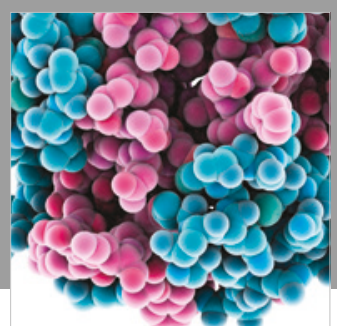

ournal of

Diabetes Research

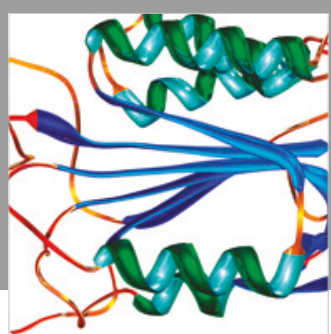

Disease Markers
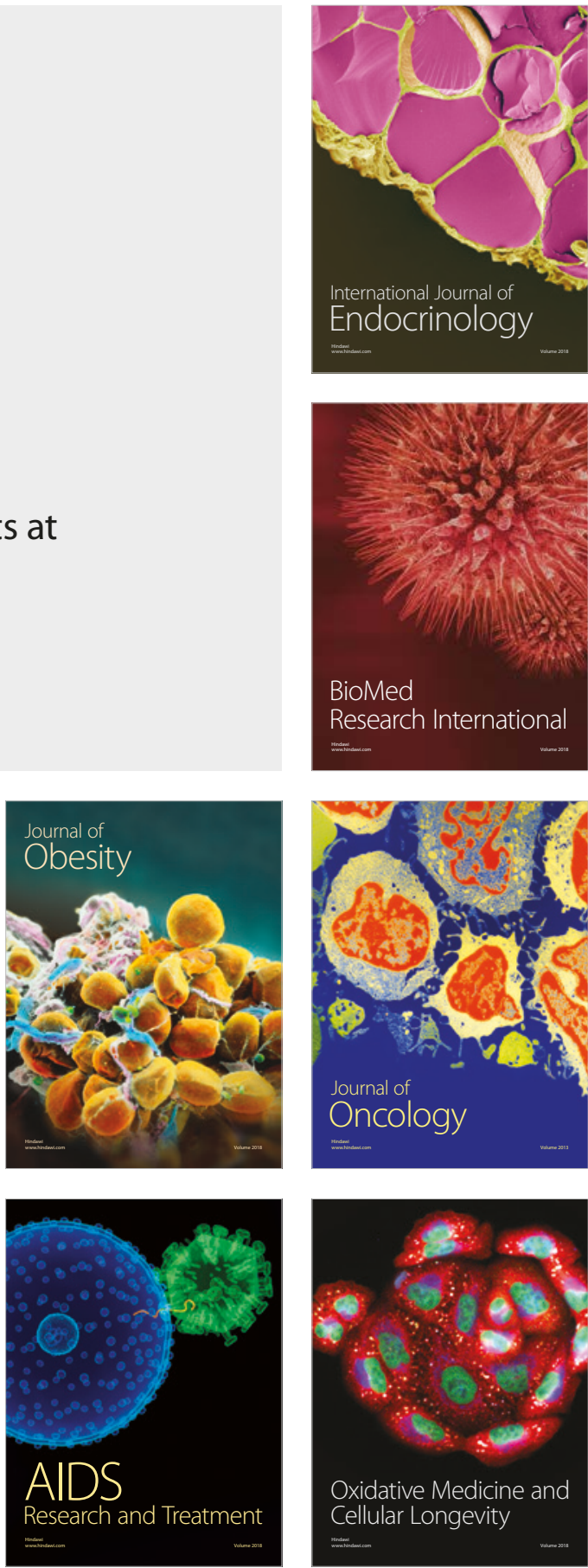